\title{
Study into the Role of Nickel Vapor on Surface Modification of a Third-Generation Single-Crystal Superalloy
}

\author{
DIMITRA SPATHARA, DUNCAN PUTMAN, and NILS WARNKEN
}

\begin{abstract}
A third-generation single-crystal (SX) superalloy was heat treated at $1350{ }^{\circ} \mathrm{C}$ for 24 hours while being exposed to nickel vapor. It is observed that a layer of $60-\mu \mathrm{m}$ thickness deposited onto the alloy. This prevented the formation of TCP phases and the occurrence of melting, which were reported in previous studies. The interdiffusion between the alloy and deposited layer shows strong indications of cross-diffusion effects, as shown from the composition profile measurements and simulations.
\end{abstract}

https://doi.org/10.1007/s11661-018-4712-2

(c) The Author(s) 2018

\section{INTRODUCTION}

SINGLE-CRYSTAL (SX) nickel-based superalloys, due to their superior resistance in creep and fatigue properties, have undoubtedly contributed to the impressive evolution of energy efficient modern jet engines. ${ }^{[1-4]}$ Industrial processing involves casting followed by solution heat treatment, during which pronounced surface modification has been reported for the Re-rich third-generation Ni-based superalloys. ${ }^{[5]}$ It was suggested that this could be the result of sublimation - the direct transition from solid into the gas state - of alloying elements at high temperature and high vacuum. The idea that a Ni source can act as an inhibitor to the reaction of sublimation was first suggested by Wang et al. ${ }^{[5]}$ The current work aims to explore this idea further and study the effect on the microstructure beneath the surface of a third-generation SX superalloy after it has been exposed to Ni vapor at high temperature. Simulations are used to rationalize the observations.

\section{EXPERIMENTAL AND SIMULATION METHODS}

\section{A. Heat Treatment Experiments}

Experiments were performed to allow $\mathrm{Ni}$ to sublimate from a source of pure $\mathrm{Ni}$ and to resublimate on a sample of SX superalloy. This used the fact that the

DIMITRA SPATHARA and NILS WARNKEN are with the School of Metallurgy and Materials, University of Birmingham, Edgbaston, Birmingham B15 2TT, UK. Contact e-mail: n.warnken@bham.ac.uk DUNCAN PUTMAN is with the Global Manufacturing Centre, Rolls-Royce plc., Derby DE24 8BJ, UK.

Manuscript submitted March 15, 2018.

Article published online June 13, 2018 activity of $\mathrm{Ni}$ in the superalloy is lower than the activity in pure $\mathrm{Ni}$, which is, by definition, equal to one. The SX Ni-based superalloy CMSX-10K* has

${ }^{*}$ CMSX-10 is a registered trademark of Cannon-Muskegon Corporation, 2875 Lincoln St, Muskegon, MI.

been subjected to a 24-hour heat treatment at $1350{ }^{\circ} \mathrm{C}$, surrounded by $\mathrm{Ni}$ foil, to provide a source for $\mathrm{Ni}$ vapor, wrapped in such a way that the foil was not in contact with the surface of the alloy sample. The nominal alloy composition can be found in Table I. A cylindrical bar of $8-\mathrm{mm}$ diameter and $65-\mathrm{mm}$ length, already solution heat treated and primary aged, was polished mechanically by surface reduction of around $0.5 \mathrm{~mm}$ to eliminate any modification that may have been present from previous processing. Subsequently, the sample was cross sectioned along the long dimension by electrical discharge machining. A section of $10-\mathrm{mm}$ length was cut using a $\mathrm{SiC}$ cutting disc. It was then placed on an alumina boat, inside a high vacuum furnace. Alumina tubes of $10-\mathrm{mm}$ diameter were placed on each side of the alumina boat, to allow wrapping the $\mathrm{Ni}$ foil without contact between the foil and sample. Three layers of $\mathrm{Ni}$ foil, around $20-\mathrm{cm}$ wide, surrounded the alumina boat and alumina tubes inside the furnace. The heat treatment was performed in high vacuum, under 0.2-mbar Ar pressure, using a heating rate of $20 \mathrm{~K} / \mathrm{min}$ to $1350{ }^{\circ} \mathrm{C}$, followed by an isothermal hold of 24 hours. The sample was then quenched as fast as possible, using the same configuration as described in Reference 6, consisting of pressurizing the furnace using argon to 10 bar and circulating the gas through a heat exchanger. 
Table I. Composition of the Studied Alloy CMSX-10K (Weight Percent) ${ }^{[7]}$

\begin{tabular}{lcccccccccc}
\hline Weight Percent & $\mathrm{Al}$ & $\mathrm{Ti}$ & $\mathrm{Cr}$ & $\mathrm{Co}$ & $\mathrm{Mo}$ & $\mathrm{Nb}$ & $\mathrm{Ta}$ & $\mathrm{W}$ & $\mathrm{Re}$ & $\mathrm{Ni}$ \\
\hline CMSX-10K & 5.8 & 0.2 & 2.3 & 3.3 & 0.4 & 0.1 & 8.3 & 5.5 & 6.3 & bal \\
\hline
\end{tabular}

The cross section of the sample was then examined by cutting a section of 5-mm length followed by mounting, grinding, polishing, and fine polishing. The layer beneath the surface was studied using scanning electron microscopy (SEM) and backscattered electron imaging (BEI). Composition profiles were measured using energy-dispersive X-ray (EDX), starting from the surface and extending about $530 \mu \mathrm{m}$ into the sample, with a point measurement every $5 \mu \mathrm{m}$. Additional measurements took place parallel to the surface at various locations. Finally, electron backscatter diffraction (EBSD) mapping was carried out. SEM, BEI, EDX, and EBSD were performed with the field emission gun-scanning electron microscope JEOL** $7000 \mathrm{~F}$.

**JEOL is a trademark of Japan Electron Optics Ltd., Tokyo.

B. Modeling the Interaction Between the Substrate and the Deposited Layer

DICTRA $^{[8]}$ simulations were used to simulate the interaction between the deposited Ni layer and the alloy at $1350{ }^{\circ} \mathrm{C}$. The domain was selected to be of $700 \mu \mathrm{m}$, and 700 grid points were entered linearly across the domain. The thermodynamic and kinetic database TTNI8 and MOBNI1 were used, respectively. A labyrinth factor was used to include the $\gamma^{\prime}$ phase, as discussed in a previous work. ${ }^{[9]}$ At the chosen temperature, however, very little, if any, formation of $\gamma^{\prime}$ phase is expected. As the deposition of Ni cannot be simulated in DICTRA, the experiment was approximated in two ways: by (a) single-layer and (b) multilayer simulations. In the first case, it is assumed that a layer of $60-\mu \mathrm{m}$ thickness of pure $\mathrm{Ni}$ formed on the surface of the superalloy sample. The interdiffusion between the $\mathrm{Ni}$ layer of $60-\mu \mathrm{m}$ thickness and a layer of CMSX-10K of $640-\mu \mathrm{m}$ thickness was then simulated. The dimension of the Ni layer was chosen in line with the experimentally observed layer thickness. In the case of the multilayer approach, the simulation was refined by gradually building up the Ni layer during the simulation. It was assumed that $1 \mu \mathrm{m}$ of $\mathrm{Ni}$ was deposited every $24 \mathrm{~min}-$ utes, in total 60 layers within 24 hours of heat treatment. The DICTRA grid was, accordingly, extended by additional grid points containing pure Ni. This layer would then interdiffuse with the existing material before an extra layer would be added, and so on. Adding new grid points is not possible inside DICTRA, however. This limitation was overcome by stopping simulations, writing composition profiles to files, and altering the profiles as described previously, before restarting the simulation. A small, dedicated software tool was written for this purpose.

\section{RESULTS AND DISCUSSION}

After the heat treatment, the surface of the sample was found to be shiny with only some light gray marks in the areas of the alumina tubes. The alumina boat showed very few hints of metal deposition. BEI depicted that after a 24-hour heat treatment in the presence of the $\mathrm{Ni}$ source at $1350^{\circ} \mathrm{C}$, there was no evidence of surface melting or TCP formation as found in previous work. ${ }^{[5]}$ On close inspection using BEI and EBSD mapping, different layers were distinguished near the surface of the sample (Figure 1). The most obvious one was a thin layer of pores, which marked the original surface of the sample. This layer separated the SX material and a polycrystalline layer above. EDX point measurements around the pores showed areas rich in $\mathrm{Ni}, \mathrm{Al}$, and $\mathrm{O}$, with 70 at. pet Ni, 13 to 14 at. pet Al, and 12 to 13 at. pet $\mathrm{O}$. The layer above the porous zone consisted of grains with different orientations. This outer layer of around $60-\mu \mathrm{m}$ thickness was considered to be the deposited layer above the initial surface of the alloy. Naively, it could be expected that this layer would be, to a large extent, pure Ni. However, the EDS data shows significant levels of all alloying elements, indicating significant levels of interdiffusion between deposited $\mathrm{Ni}$ and the alloy. This will be looked at in more detail when the simulation results are compared (Figure 3).

The results of simulation of interdiffusion between the $60-\mu \mathrm{m}$ layer of pure $\mathrm{Ni}$ and CMSX-10K for 24 hours at $1350{ }^{\circ} \mathrm{C}$ are shown in Figure 2. The composition profiles for various times during the heat treatment are shown. Simulations were preformed in the volume fix frame of reference, using $\mathrm{Ni}$ as the dependent composition. The Ni profile in Figure 2 shows a significant drop of the Ni composition over time; the profiles do not show any unusual features. All other elements, except $\mathrm{Cr}$, show a strong indication of interaction of the diffusing species; Al, Ta, and, to a lesser extent, Co show local minima and maxima close to the initial joint between the alloy of the Ni layer, while $\mathrm{W}$ and Re show an initial increase above the alloy composition at the same location. Toward the end of the 24 hours, most of these disappear or become less pronounced, except for $\mathrm{Al}$, which still shows a very peculiar profile. The Al profile shows the nominal composition at the alloy bulk $(700 \mu \mathrm{m})$ at the surface of the sample $(0 \mu \mathrm{m})$. In between, a deep dip in composition can be seen, whose existence would be difficult to understand without knowing the temporal evolution.

Simulated and measured composition profiles are shown in Figure 3. Here, simulations where the Ni layer was gradually built up as 60 individual layers are also included (multilayer results). This was intended to test if this would be a better approximation to the deposition 

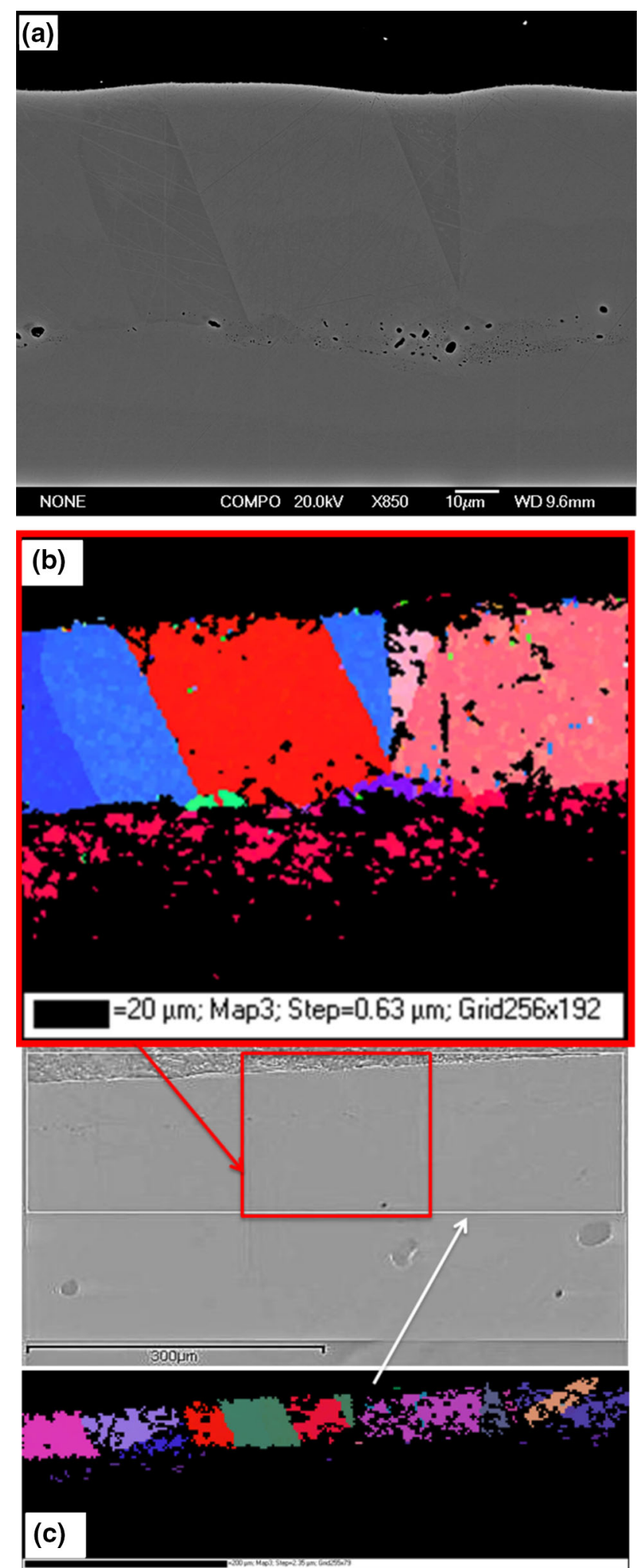

Fig. 1-(a) Magnification 850 times BEI of the surface, showing a thin porous zone separating large grains at the deposited, upper layer from a more homogeneous microstructure toward the bulk. (b) EBSD mapping performed at the same frame as in (a), demonstrating the formation of large grains at the surface. (c) EBSD mapping at a larger area, showing grain spanning the deposited layer. Scale bar: $200 \mu \mathrm{m}$, step $=2.35 \mu \mathrm{m}$, and Grid $255 \times 79$. process. It can be seen that the single-layer and multilayer results qualitatively are very similar, reproducing the same overall trends. For the multilayer simulation, all elements, except $\mathrm{Ni}$, which is the dependent composition, show higher compositions in the deposited layer than in the single-layer simulation. The multilayer simulation effectively decreases the diffusion distance, as the first layers deposited quickly saturate before new layers are added. This also effectively accelerates diffusion into the deposited material, as steep composition gradients are maintained throughout the process.

When compared to the measured composition profiles, the simulation results show higher compositions in the deposited layer for the elements $\mathrm{Al}, \mathrm{Cr}, \mathrm{Co}$, and $\mathrm{Ni}$, while Re, Ta, and W show lower ones. From the surface of the sample toward the bulk, the elements $\mathrm{Cr}, \mathrm{Co}, \mathrm{Re}$, and $\mathrm{W}$ show a steady increase in composition, while $\mathrm{Ni}$ shows a drop. Al also shows a drop, but the simulations show a local minimum, while measured values stay below the nominal composition. Ta shows a decrease in the experiments but an increase in the simulations, with a local maximum. The measured Ta composition at the surface even lies above the nominal composition. The multilayer simulation approach gives a Ta composition closer to the measurement, except for the point $50 \mu \mathrm{m}$ below the surface. Generally, both simulation approaches reproduce the trends seen in the measurement for all elements, except for $\mathrm{Al}$ and $\mathrm{Ta}$, but the absolute compositions are not always correct. This clearly indicates that an important effect was not taken into account in the simulations. This will be discussed in detail in Section IV.

Figure 4 shows the activity of $\mathrm{Ni}$ at the surface of the alloy as a function of time, as calculated from the single-layer and multilayer simulation. The horizontal dashed lines indicate the activities of pure $\mathrm{Ni}$ in the $\mathrm{Ni}$ foil, and in the alloy prior to any deposition. The difference of activities between the foil and sample surface is proportional to the driving for Ni to sublimate and deposit. As the deposited layer in the multilayer simulation is initially very thin, diffusion from the alloy is sufficient to change the layer composition. Thus, the initial $\mathrm{Ni}$ activity in this case is close to the one in the alloy and gradually increases as more $\mathrm{Ni}$ deposits. In the single-layer case, the surface of the material is initially pure $\mathrm{Ni}$; therefore, the $\mathrm{Ni}$ activity is one. During the simulation, the composition of the deposited layer changes, as seen in Figure 2, lowering the activity of $\mathrm{Ni}$ on the surface. It becomes apparent that these are thermodynamically different cases. During the multilayer simulation, the deposition rate would decrease with time, while in the single-layer case, it would increase. Activities in both cases do converge, however, to the same value. The crossed symbol at 24 hours indicates the calculated $\mathrm{Ni}$ activity for the measured composition closest to the surface. The value is very close to the simulation results. Other elements in the vapor would only have a small effect, as these would affect the total pressure and diffusion rate of $\mathrm{Ni}$ in the gas but not the partial pressure of $\mathrm{Ni}$. 

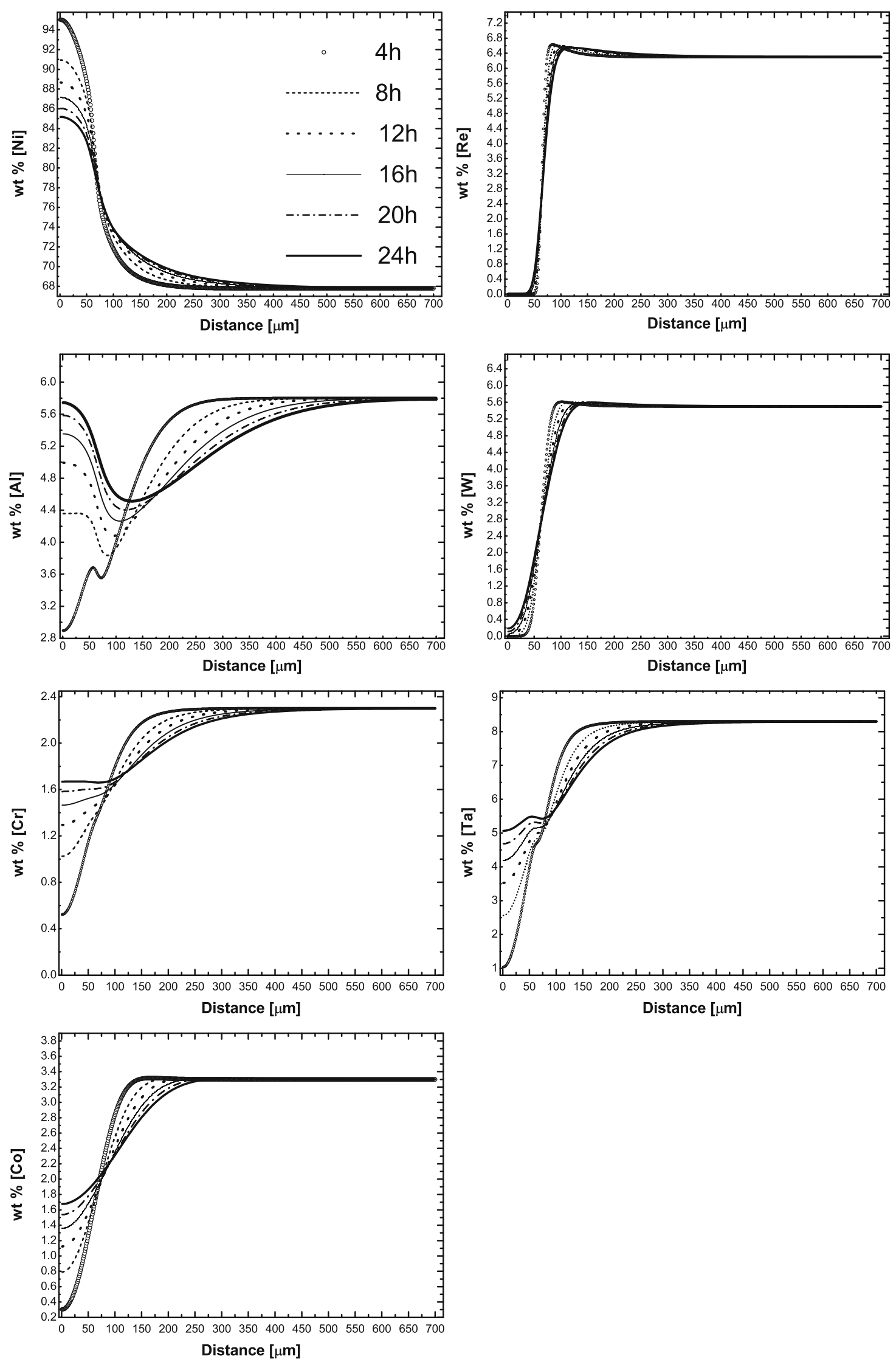

Fig. 2-Composition profiles obtained from DICTRA simulations of interdiffusion between a $60-\mu \mathrm{m}$ layer of pure Ni on CMSX-10K, $24 \mathrm{~h}$ at $1350{ }^{\circ} \mathrm{C}$. 

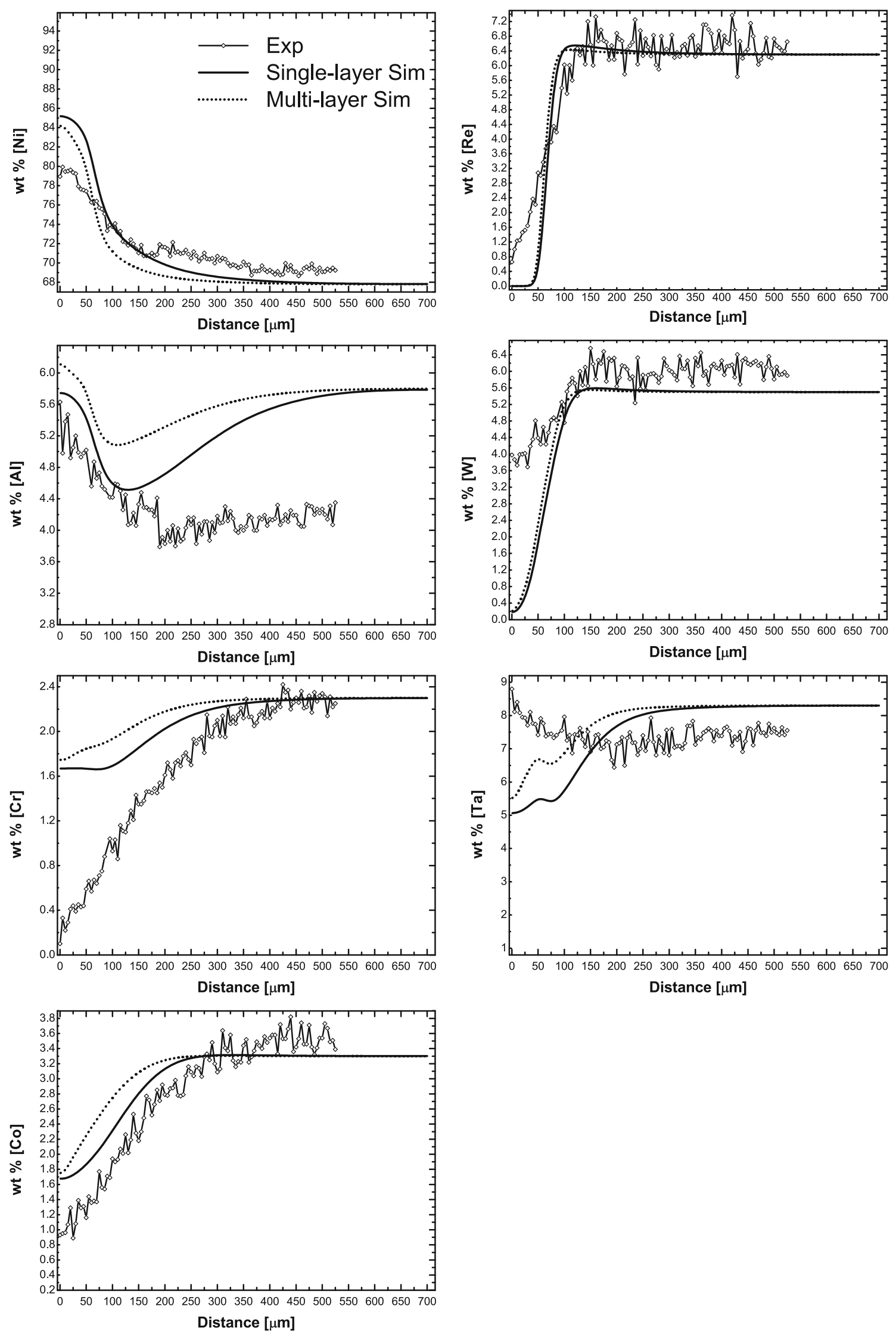

Fig. 3 - Comparison of composition profiles from EDS measurement, single-layer and multilayer simulation, after holding for $24 \mathrm{~h}$ at $1350{ }^{\circ} \mathrm{C}$. 


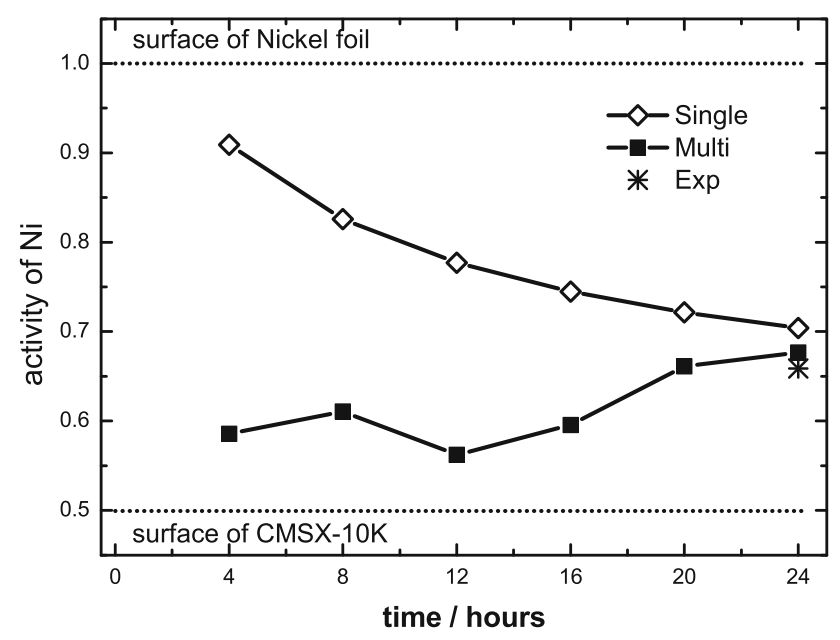

Fig. 4-Evolution of $\mathrm{Ni}$ activity as obtained from single-layer and multilayer simulation in comparison to pure $\mathrm{Ni}$ foil and unaltered alloy, during holding for $24 \mathrm{~h}$ at $1350{ }^{\circ} \mathrm{C}$.

\section{DISCUSSION}

The results clearly show that Ni sublimated from the foil and deposited on the alloy. This can be explained by looking at the thermodynamics of the problem. The activity of $\mathrm{Ni}$ in the foil is equal to unity and considerably higher than that in the alloy. For the pure $\mathrm{Ni}$ to be in equilibrium with the gas phase, the Ni partial pressure in the gas has to be higher than it is in the gas over the alloy. Hence, $\mathrm{Ni}$ will sublimate from the Ni foil and deposit on the alloy, providing a source and a sink of $\mathrm{Ni}$.

As the added Ni layer interdiffuses with the alloy, its composition evolves toward the alloy composition, while the alloy gets diluted by the additional $\mathrm{Ni}$. In both cases, the refractory element content is more diluted, lowering the probability of forming TCP phases, while reducing the $\mathrm{Al}$ content mitigates the risk of melting. It is, thus, interesting to consider if this technique can be used to mitigate risks of surface melting and TCP formation during solution heat treatments. It is often observed that the outermost turbine blades in a heat treatment furnace load show the most severe damage. This indicates that sublimation from heat-treated parts creates a Ni-rich atmosphere that is almost enough to prevent damage. Adding a sacrificial Ni part, at the outer parts, has the potential to reduce or event prevent sublimation-induced damage. Furnace operators might be concerned about the additional $\mathrm{Ni}$ vapor, which will eventually deposit elsewhere in the furnace. This vapor is there even without sacrificial $\mathrm{Ni}$, sublimating from heat-treated alloys and, hence, damaging them.

The composition profiles observed in the experiments look surprising at first glance, especially when compared to the simulation results. It is clear that the high temperature $\left(1350^{\circ} \mathrm{C}\right)$ sufficiently accelerates diffusion to enable noticeable composition change in the deposited layer. As the layer is relatively thin $(60 \mu \mathrm{m})$, the entire deposited layer is affected by interdiffusion. Cross-diffusion effects are very noticeable in the composition profiles of most elements, either as local minima/maxima (Al, Ta, Cr) or as overshoots in composition (Re, W). These occur when the gradient in one element drives the diffusion in another and are quite common in multicomponent alloys, especially when the composition difference is rather large. In the case of $\mathrm{Al}$, cross-diffusion is also the reason for the composition profile observed in both experiments and simulation. In both cases, high concentrations are observed on the surface of the sample. Al is driven toward the surface by gradients of other elements; it is, however, not possible to determine without further analysis which elements are the main driving forces.

Another important observation is that the measured composition of some elements at the surface is higher than calculated ( $\mathrm{Re}, \mathrm{W}, \mathrm{Ta}, \mathrm{Ni}$ ), while for other elements, the reverse is true ( $\mathrm{Al}, \mathrm{Co}, \mathrm{Cr})$. The elements $\mathrm{Al}, \mathrm{Co}, \mathrm{Cr}$, and $\mathrm{Ni}$ have significantly higher vapor pressures than the other elements. ${ }^{[10]}$ In a previous study, it was suggested that these elements sublime during heat treatments. ${ }^{[5]}$ The vapor above the sample is rich in $\mathrm{Ni}$, due to the presence of a $\mathrm{Ni}$ source, but poor in $\mathrm{Al}, \mathrm{Cr}$, and Co. The latter elements, therefore, experience a driving force to sublimate and increase their partial pressure in the vapor. Consequently, the alloy will lose some of these elements. Even Ni will sublime from the alloy during the early stages of the experiment, while the Ni partial pressure builds up. In the case of $\mathrm{Al}$, cross-diffusion drives $\mathrm{Al}$ to the surface, as gradients in composition of other elements drive $\mathrm{Al}$ diffusion. $\mathrm{Al}$ is the fastest diffusion element in the alloy and will, therefore, reach the surface first. This might also explain why the measured $\mathrm{Al}$ composition does not recover to the nominal $\mathrm{Al}$ composition, as sublimation started from the earliest stages of the experiment. As Al, $\mathrm{Cr}$, and Co are lost, the relative compositions of Re, W, and Ta will increase, as they are left behind. This explains why the measured compositions of these elements are higher than the simulated ones. The difference between experimental and simulated results for $\mathrm{Ta}$ is quite significant, though. The phenomenon explained previously, plus the cross-diffusion effect seen in the simulation, might explain most of the difference, but it should also be considered that the thermodynamic and kinetic data are not as accurate as they should be. Further investigations are needed to clarify this. However, most of the difference between the experiment and simulation is due to the fact that the model assumes a closed system, while the alloy in the experiment is an open system with exchange of matter between gas and solid phases.

The assumptions made in the simulations with regard to the kinetics of $\mathrm{Ni}$ deposition are quite simple: all the $\mathrm{Ni}$ is deposited either instantly or at a constant deposition rate. The reality, of course, is more complex, as the deposition rate depends on the actual partial 
pressure of $\mathrm{Ni}$ in the gas phase and the actual activity of $\mathrm{Ni}$ on the surface of the alloy. As the Ni layers deposit and the alloying elements interdiffuse, the $\mathrm{Ni}$ activity changes and, hence, the deposition rate changes. The assumption of constant deposition, therefore, is not accurate, but it does, however, allow a qualitative estimate of the effect of noninstantaneous deposition, which is an increase of diffusion into the deposited layer, resulting in higher compositions of all alloying elements.

Finally, the rather large composition differences between the alloy and deposited layer can lead to nonbalanced diffusion fluxes. In the present case, more alloying elements diffuse into the deposited layer that $\mathrm{Ni}$ diffuses into the alloy, and the difference in volume shows up as porosity, commonly referred to as Kirkendall porosity. However, this does not explain the enrichment of some alloying elements around the pores. Further investigations are needed to confirm this in the present case.

\section{CONCLUSIONS}

1. Heat treatment of CMSX-10K in the presence of pure Ni was performed and DICTRA simulations were used to rationalize the observed composition profiles.

2. It was found that a considerable amount of $\mathrm{Ni}$ was deposited onto the CMSX-10K sample. The deposited material was polycrystalline and, interestingly, the composition was not pure $\mathrm{Ni}$ but contained considerable amounts of all alloying elements of CMSX-10K. Furthermore, no TCP phases or melting of the alloy were observed. The compositional change of the deposited layer was explained by the interdiffusion between the alloy and deposited layer and simultaneous sublimation of $\mathrm{Al}, \mathrm{Cr}$, and $\mathrm{Co}$.

3. The results show that by controlling the gas atmosphere, the reaction between the gas/vapor and alloy can be reversed from the loss of $\mathrm{Ni}$ to the deposition of Ni. The observed structures are fundamentally different from structures seen without the presence of pure Ni. This also supports the sublimation hypothesis set up in previous publications.

\section{ACKNOWLEDGMENTS}

The authors thank Dr. Bo Pang for helping with the EBSD mapping and Mr. Peter Cranmer for carrying out the heat treatment at the high vacuum furnace. SEM, EDX, and EBSD studies took place at the Electron Microscopy Group Facility, University of Birmingham. The financial support of this work was provided by Rolls-Royce plc and Innovate UK.

\section{OPEN ACCESS}

This article is distributed under the terms of the Creative Commons Attribution 4.0 International License (http://creativecommons.org/licenses/by/4.0/), which permits unrestricted use, distribution, and reproduction in any medium, provided you give appropriate credit to the original author(s) and the source, provide a link to the Creative Commons license, and indicate if changes were made.

\section{REFERENCES}

1. R.C. Reed: The Superalloys: Fundamentals and Applications, Cambridge University Press, Cambridge, 2006.

2. C.T. Sims: Superalloys II, C.T. Sims, S.N. Stoloff, and C.W. Hagel, eds., Wiley, New York, NY, 1987, pp. 3-26.

3. T.M. Pollock and S. Tin: J. Propul. Power, 2006, vol. 22 (2), pp. 361-74.

4. F.I. Versnyder and M.E. Shank: Mater. Sci. Eng., 1970, vol. 6 (4), pp. 213-47.

5. H. Wang, N. D'Souza, S. Zhao, D. Welton, N. Warnken, and R.C. Reed: Scripta Mater., 2014, vol. 78, pp. 45-48.

6. F. Cosentino, J.-C. Gebelin, N. Warnken, and R.C. Reed: 9th Int. Conf. on CFD in the Minerals and Process Industries, 2012, C.B. Solnordal, P. Liovic, G.W. Delaney, and P.J. Witt, eds., CSIRO, Melbourne, http://www.cfd.com.au/cfd_conf12/PDFs/031COS.pdf, accessed March 14, 2018.

7. N. D'Souza and H.B. Dong: Superalloys 2008, R.C. Reed, K.A. Green, P. Caron, T. Gabb, M.G. Fahrmann, E.S. Huron, and S.A. Woodard, eds., TMS, Warrendale, PA, 2008, pp. 261-69.

8. J.-O. Andersson, T. Helander, L. Höglund, P. Shi, and B. Sundman: Calphad, 2002, vol. 26, pp. 273-312.

9. M. Bensch, A. Sato, N. Warnken, E. Affeldt, R.C. Reed, and U. Glatzel: Acta Mater., 2012, vol. 60 (15), pp. 5468-80.

10. C.B. Alcock, V.P. Itkin, and M.K. Horrigan: Can. Metall. Q., 1984, vol. 23, pp. 309-13. 